\title{
A Developmental Psychobiological Approach to Human Development
}

By: George F. Michel

Michel, G.F. (2014). A Developmental Psychobiological Approach to Human Development. Research in Human Development, 11(1), 37-49. doi: 10.1080/15427609.2014.874764

This is an Accepted Manuscript of an article published by Taylor \& Francis in Research in Human Development on 05 Feb 2014, available online: http://wwww.tandfonline.com/10.1080/15427609.2014.874764.

***(C) Taylor \& Francis. Reprinted with permission. No further reproduction is authorized without written permission from Taylor \& Francis. This version of the document is not the version of record. Figures and/or pictures may be missing from this format of the document. $* * *$

\begin{abstract}
:
Individuals inherit not just genes from the parents but also an epigenetic pattern, an ecological (social, biological, and physical) niche, and perceptual and behavioral biases (e.g., culture), all of which can provide experiences necessary for the formation of nascent systems upon which subsequent learning and development depend. Some models of development are evaluated and the developmental psychobiology model is presented as the best way of elucidating how certain early experiences can shape nascent systems that will contribute to the development of a wide range of social, emotional, and cognitive abilities in humans. An example from handedness development is used to illustrate the model.
\end{abstract}

Keywords: Developmental psychobiology | Human development | Genetics | Handedness

\section{Article:}

Let me begin with a query that appeared on a social media website directed toward researchers. "Are right-/left-handers a gene-determined allocation or a learned habit? For either answer you'd prefer, why is the ratio of distributions not even?” (ResearchGate.net, September 27, 2013). Since I have investigated the development of human handedness (especially during infancy) for the past 40 years, I was surprised because the query's first question illustrates a lack of conceptual progress during the last 100 years. The second question about the ratio of the distribution begs a developmental answer that is not readily accommodated by the two alternatives proposed in the first question.

So, in part, this article addresses the question: How do we get people to stop posing the first question? I do not have a ready answer to that question, but I will use some examples from my research on the development of human handedness to illustrate how early perceptual and behavioral asymmetrical biases can provide experiences relevant to the formation of the nascent 
sensorimotor systems upon which the development of handedness progresses. These nascent systems should not be mistaken for early evidence of either the mechanism or functioning of later developing handedness; they are simply the precursors and prerequisites upon which the development of the later handedness depends.

Most scientists readily assert (almost like a mantra) that the nature-nurture controversy is dead. Instead, they note that all traits develop from a gene-by-environment interaction (GBEI). They then propose that the research task is to demonstrate this interaction. Of course, this formulation assumes that the genes control some set of processes that are responsive to some set of environmental conditions that will affect the distribution of individual differences in the phenotypes within a population. I argue that this formulation and its assumptions fail to capture the processes responsible for the development of traits and for their potential alteration by preventive and rehabilitative intervention in the case of undesirable traits (cf. Michel, 2010b). Developmental psychobiologists (DPB) have long argued that phenotypes develop from the interaction of the characteristics of the individual (the status of physiological systems and biomechanical properties established by earlier development) with his or her environment (shaped in part by the individual's current and previous participation with his or her social and physical milieu) and the individual and the environment can change throughout the life span (Michel \& Moore, 1995).

When applied to psychological traits, GBEI research uses modern sophisticated characterizations of the genome (defined with modern molecular techniques) and the environment (defined with more details of peoples' socioeconomic status [SES] and sociological conditions). Then, computationally complex statistical techniques are applied to the distributions of the traits relative to these predictor variables and their relation. These procedures improve our prediction of atypical, undesirable, or problematic traits and they have revealed that some rather striking differences in psychological traits are associated with specific gene-environment interactions. Thus, such GBEI studies provide useful predictions about the distribution of some individual differences in the manifestation of psychological traits. However, these studies do little to unravel the processes underlying the development of the traits in question and reveal little about how to prevent the manifestation of the atypical, undesirable, or problematic trait or how to rehabilitate individuals who have developed the trait.

Using evidence from my research on handedness development (cf. Michel, Nelson, Babik, Campbell, \& Marcinowski, 2013), I illustrate that only systematic longitudinal research can identify some of the ubiquitous and nonintuitive conditions that can contribute to the development of the individual's traits. But first, I need to demonstrate the problem of GBEI research for understanding development.

GENE-BY-ENVIRONMENT INTERACTION (TRANSACTIONAL MODELS) 
Modern researchers who use the concept of GBEI to characterize the development of traits typically apply a transactional model (e.g., Tucker-Drob, Briley, \& Harden, 2013).

Transactional models posit that these gene-environment correlations are key mechanisms of cognitive development. Early genetically influenced behaviors lead a person to select (and to be selected into) particular types of environments; these environments, in turn, have causal effects on cognition and serve to reinforce the original behaviors that led to those experiences. (TuckerDrob et al., 2013, p. 350)

These models presume that people in high-opportunity contexts differentially evoke and select experiences that contribute to their developmental outcomes (often considered to be positive) primarily on the basis of their genetic predispositions. The result of such a transactional process is that the association with genetic measures increases with age and with increasing environmental opportunity. Similarly, in low-opportunity contexts, genetic predispositions evoke and select experiences that contribute to developmental outcomes (often considered to be negative) and, also, will exhibit increasing association with genetic measures with age.

Consider just one well-known study of individual variability and similarity in the expression of conduct disorders in human children that showed an association with the genotype-environment interaction (Caspi et al., 2002; Jaffee et al., 2005). Individuals who were maltreated as children were more likely to be diagnosed as adults with antisocial personality or conduct disorder if they had a polymorphism for low expression of the monoamine oxidase A (MAOA) genotype compared to those with a genotype resulting in high levels of expression of MAOA. Sometimes such research is considered as conforming to Gottlieb's (1999) illustration of the developmental manifold, see Figure 1, because it acknowledges that the relation of gene expression to manifest phenotype is a consequence of the interaction captured by Gottlieb's illustration. However, even when there is a high probability of predicting the trait, the GBEI approach fails to prompt developmental investigations of how the relation becomes predictive. This also is a failure of Gottlieb's illustration (but not his reasoning). GBEI research does not reveal:

1. The character of the developmental trajectories that led to the expressed phenotypes of individuals

\section{FIGURE 1 IS OMITTED FROM THIS FORMATTED DOCUMENT}

2. The relation of the individual's earlier appearing phenotypes to the phenotypes of interest or of the mechanisms governing the transitions (or lack thereof) in the successive expression of the developing phenotypes that comprise the trajectory that results in the trait of interest 
3. Explanations for why some individuals with both the "predisposing” polymorphism and the "predisposing”' child abuse did not become adults with antisocial "personalities"

4. Explanations for why some individuals, without either of the "predisposing" conditions, nevertheless develop an antisocial "personality” as adults?

Thus, representation of developmental trajectories and alternative pathways are missing from Gottlieb's illustration. The primary task of developmental science is to understand how the psychological repertoire of an individual develops along trajectories that start with conception and proceed across the life span via differences in the manifest forms (phenotypes) of the individual's traits and their underlying processes. This task includes identifying developmental processes that are capable of producing individual differences and species-typical similarities among psychological traits. Figure 2 illustrates how the investigation of developmental trajectories can reveal how similar starting points can diverge extensively and how dissimilar stating points can converge. The DPB task requires investigation across all of the levels of organization of the individual from cellular processes to coordination among systems as these play out in individual trajectories - the task is not easy.

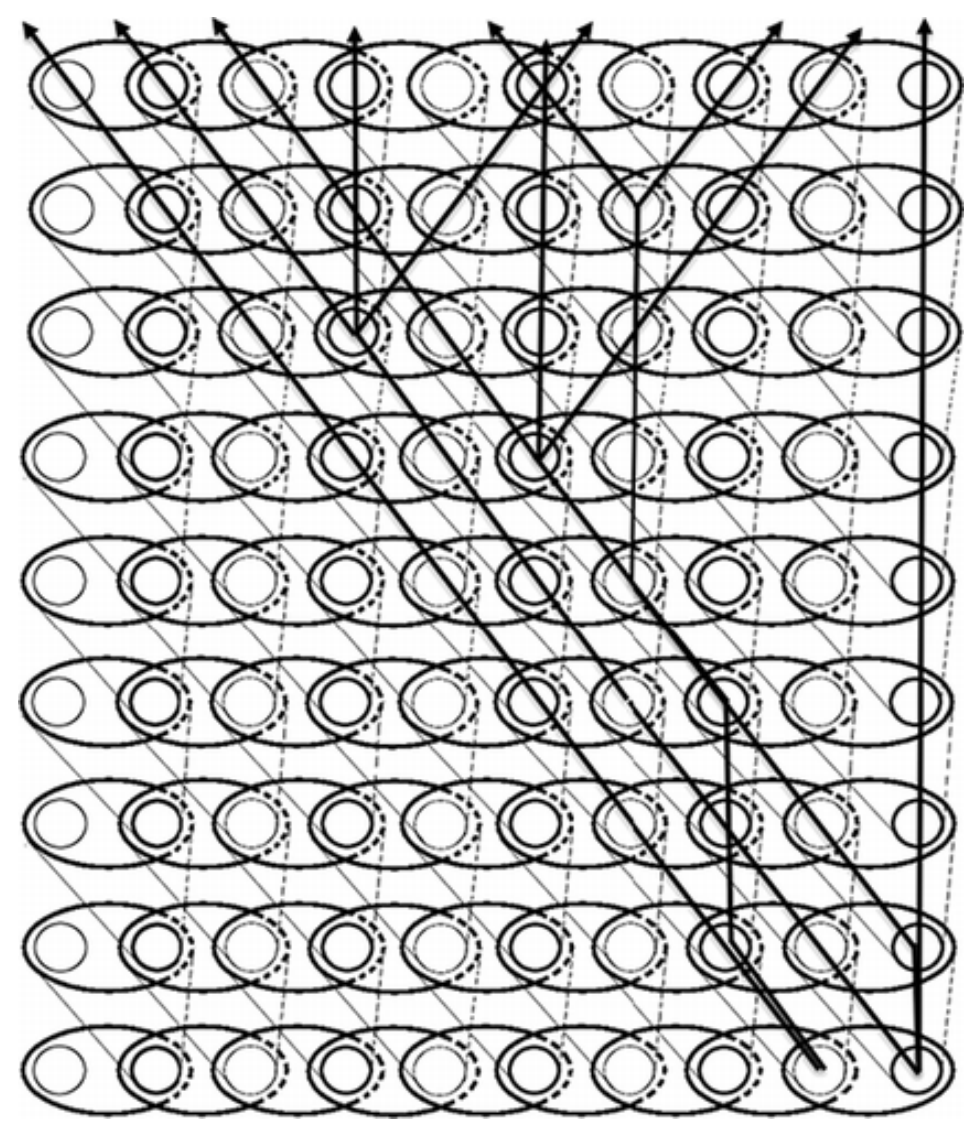


FIGURE 2. This model illustrates developmental trajectories emerging from the interaction of the individual (circles) with the immediate environment (ellipses). Darker and lighter circles represent different genomes and each level of the ellipses represents different phenotypes at different developmental time points with the ellipses representing the individual's environment at that time point. Development begins at the bottom of the figure. (C G. F. Michel (2013).

Nearly 50 years ago, Nobel Laureate in developmental neurobiology Roger Sperry (1965) provided a detailed characterization of the levels of organization and the complexity of causality that is involved when attempting to account for the actions of the nervous system.

[T] he molecules of the brain are ... obliged to submit to a course of activity in time and space that is largely determined ... by the overall dynamic and spatial properties of the whole [brain] cell ... [which does] not have very much to say about when they are going to fire their messages ... or in what time pattern they will fire them ... . The flow and timing of [neural activity is] ... governed largely by ... properties of the whole cerebral circuit, within which the given cells and fibers are incorporated, and also by the relationship of this circuit system to other circuit systems. Further ... the general circuit properties of the whole brain may undergo radical and widespread changes [as a result of] a shifting pattern of central excitation ... [opening or priming] ... one group of circuit pathways [having special properties while] ... closing, repressing, or inhibiting ... other circuit potentialities. Of course, all of the simpler ... molecular, cellular, and physiological forces remain present ... and continue to operate, ... but these lower level forces and properties have been superseded ... by those of higher levels of organization ... [and] proper function in the uppermost levels always depends on normal operation at subsidiary levels. (Sperry, 1965, p. 79)

For Sperry (1965), the activities at the lower levels of organization (e.g., neurons) are constrained and influenced by their participation in higher levels of organization (e.g., neural circuits or networks). The higher levels depend upon the proper functioning of the lower levels, but the lower levels do not dictate the functioning of the higher levels. If we add to this account of multiple levels of causal complexity in the nervous system, the relation of the nervous system to the other systems of the individual (endocrine, immunological, skeletal-muscular, etc.), then, it should be clear that the individual's behavior and psychological functioning is not simply a product of the nervous system or GBEI. Indeed, it is important to include in Sperry's hierarchy the way that the individual's behavioral functioning is conditioned, constrained, and prompted by the organized social and physical milieu of that individual. Thus, the emergence of organized patterns of behavior and psychological functioning in an individual involves the coordinated action of sensory, neuromuscular, skeletal/muscular, hormonal, and the neural processing, as well as the specific consequences of the concurrent and past social and physical milieu on that coordinated action. GBEI simply ignores the contributions of these important levels as they construct the individual's developmental trajectory. DPB seeks to include these levels, not all in the same study but rather via systematic programmatic research across studies. 
Because the individual participates in the milieu and can alter, modulate, adjust to and avoid various aspects of milieu conditions, the individual, somewhat paradoxically, can maintain certain characteristics in the presence of changing circumstances and change certain characteristics in the presence of relatively unchanging milieu conditions. This has to be captured in any model of development and will likely involve nonlinear trajectories. The model also must be able to represent how the individual's behavior contributes to the condition and state of every one of the individual's physiological subsystems via the sensory and experiential feedback provided by the performance of the behavior and the consequences of the performance on the social and physical milieu. The model must permit psychological functioning to be dependent on lower level physiological conditions and events and a contributing cause of such conditions and events.

Consequently, it is not surprising that knowledge about some aspect of an individual's DNA structure, combined with knowledge about some aspect of the individual's status in society, would capture some of the complexity of that causality. Also, although the transactional model of GBEI research captures some complexity of developmental causality, it cannot define and clarify the nature of the reciprocal causal relations as they exist among the different levels of organization within the individual or between the individual and the social and physical milieu. Moreover, such an interaction cannot reveal how these different levels of causality contribute to developmental stability and developmental change in the individual's psychological traits and functioning. Such clarification can only come from systematic developmental investigations that examine the individual's biological systems in relation to his and her physical and social milieu.

Perhaps surprisingly, modern molecular genetics provided the evidence that challenged many of the assumptions about genes inherent in the GBEI accounts of psychological traits (cf. Michel, 2010b). As Sperry (1965) predicted, molecular biology revealed that the DNA (the instantiation of the gene construct) is part of a complex system (network) of causes that operates throughout the life span. The DNA does not contain specific blueprints for, or a code for, the development of any phenotypic trait (protein, morphological, or psychological). Moreover, although we inherit our DNA from our parents, we, also, inherit much of our physical and social milieu including diet, experiences, habitat (including contamination), and so on.

Modern molecular genetics revealed how the inherited environment can alter the expression of specific aspects of the DNA throughout the life span and how these influences can be transmitted to offspring through non-DNA (epigenetic) inheritance. Thus, the cell nucleus is a "chemical manufacturing plant” controlled by an intricate and dynamic set of chemical messengers that travel within and between cells to alter the timing and expression of specific aspects of DNA. This layer of biochemical reactions that affects the timing and pattern of expression of DNA involved in protein formation is called the "epigenome." Although it plays an important part in development, heredity, and health, it is not the blueprint for, code for, or director of development, heredity, or health. 
Because the epigenome can change according to an individual's environment during development and can be passed from generation to generation, the constructs of genotype ("genomic codes for traits”) and phenotype ("traits actually observed”) have been extensively revised. Traits now are considered as emerging from developmental processes that include, but are not limited to, and can be independent of, genomic action. This has led to a "revolutionary" perspective on heredity that challenges many of the assumptions that underlie neo-Darwinian theory. Instead of evolution being the consequence of changes in gene frequencies, evolution now becomes the consequence of changes in the frequencies of alternative variations in developmental trajectories derived from differences in developmental causes among members of a population (Gilbert \& Epel, 2009). Although the DNA expression is part of the network of developmental causes that affect the trajectories for the manifestation of traits throughout the lifespan, it does not control those trajectories.

But how can developmental processes achieve individuality and species typicality? Developmental psychobiological research must identify how individual differences in developmental processes are organized in such a way as to ensure a degree of continuity in development and the development of properties common across individuals. It is small wonder that the neo-Darwinian synthesis chose the simple explanations of genetic commonality for the control of species typicality and lack of genetic commonality for individual differences. Unfortunately, the choice was not supported by the evidence.

\section{WHAT IS DEVELOPMENTAL PSYCHOBIOLOGY?}

Developmental psychobiological research assumes that behavior develops from the interaction of the organism (composed of multiple levels of causal organization as described above) and its physical and social milieu. However, it adds the recognition that the individual and the milieu can change as a result of the interaction. This should not be confused with the transactional model of GBEI. Developmental psychobiological studies demonstrate the reciprocal impact of developmental factors typically described within a physiological framework (e.g., hormones, neurotransmitters, enzymes, and proteins) and developmental factors typically described within a psychological framework of social, cognitive, and emotional factors (e.g., peer influences, mnemonic strategies, "stressors") on the developmental progress of individual psychological traits (e.g., mating preferences, aggression, problem-solving skills, language, care-giving behaviors, memory capacity, altruism). The notion of genetic predisposition is an inappropriate attribution of developmental control to genes and an empirically unsubstantiated explanation of which factors affect developmental trajectories. The DNA is only a contributing factor in the functioning of the individual's physiological systems and experience can only influence those systems to the degree that they are transduced by sensory systems into physiological processes (Michel, 2010b).

DPB research provides evidence that the activation of the genome is intertwined with ecological influences in different ways at different periods throughout an individual's life span (Michel, 
2010b). Thus, to understand individual and species-typical developmental trajectories, research has to determine exactly how the environment interpenetrates with the individual's characteristics throughout the life span. This process of organism-environment interpenetration produces the characteristics expressed as the successive phases of the developmental trajectory. Moreover, the focus of the research is on understanding the development of those behaviors and psychological abilities that are manifested in the habitat typical for that species. Thus, evidence from "lab" research always must have a large degree of validity with evidence from "field" research (cf. Michel, 2010a;Michel \& Moore, 1995). This becomes problematic for humans, because their habitat is primarily socially defined.

Because developmental phenomena exist simultaneously at many levels of description (cells, tissue, organ systems, individual, family, cultural subgroup, culture, etc.), a complete explanation of any developmental phenomenon would require: (1) descriptions at all these levels without giving primacy to any one level, (2) a set of rules that would allow the interpretation of these descriptions across levels, and (3) A means of characterizing downward as well as upward influence among these levels. These three requirements make DPB research very challenging and daunting. Of course no developmental study would be able to meet all three of these requirements. However, as long as DPB research is conducted in a programmatic way with each study contributing to the understanding of the phenomenon of interest, significant progress can be made. Moreover, the DPB framework avoids disciplinary reductionism. The DPB need not give primacy of explanation to genes or the nervous system over social and cultural conditions. Also, the interaction out of which development occurs is between the properties of the individual in interaction with the properties of that individual's social and physical milieu as these operate across the life span.

Developmental science shows that, at least for humans, a symbol-based (e.g., language) inheritance also plays a substantial role in development (and evolution) via its impact on the construction and transmission of cultural, tribal, and familial traditions, routines, and habits. Indeed, humans engage in more explicit and deliberate teaching of the young than any other species and language is a major factor in such education. Therefore, symbolic inheritances must be added to the contributions to the variation in development (Jablonka \& Lamb, 2005).

The DPB approach to research considers that there are, at least, four developmental questions about psychological phenomena that require answers:

1. What are the developmental trajectories that result in the expression of the psychological trait of interest?

2. What factors facilitate and what factors constrain the individual's progression along such trajectories? 
3. What alterations of these factors produce unusual trajectories leading to new traits?

4. What alterations of these factors can reinstate a trajectory to a more typical trait?

Characterizing development as a trajectory permits comparison of trajectories for the identification of (1) those different pathways that lead to some commonality at a particular later phase of development (equifinality), (2) those pathways that reveal different phenotypes at all phases of development, (3) those pathways that begin with a set of early common phenotypes but diverge sometime thereafter, and (4) those fluctuating pathways that frequently converge and diverge during development. Understanding how differences in traits emerge resides more in knowing the difference in their developmental trajectories than in being able to predict their general outcome from some early measure.

\section{EARLY DEVELOPMENT OF HANDEDNESS}

My colleagues and I have tried to apply the DPB approach to the study of the development of human handedness. Descriptions of handedness for children and adults depend on various measures including those of speed and accuracy of timed repetitive movements (e.g., pegmoving, dotting rows of circles), relative influence of each hand in controlling bimanual actions (e.g., "etch-a-sketch” drawing, alternating hand finger-tapping rhythms), pantomimed or verbalized expressions of the hand used for unimanual or bimanual tool use (e.g., hammering), or the score on a questionnaire (the most frequently used measure). All of these measures reveal a special quality of handedness - the scores are continuously distributed across individuals with a majority with scores on the right-handedness portion of the continuum and a minority with lefthandedness scores. Also, there is a portion of people on the continuum who are neither clearly right-handed nor left-handed. Although there can be significant correlations among these measures, they are far from equivalent. This suggests that they represent different aspects of the construct "handedness," The developmental processes that contribute to the development of speed and accuracy of relative hand movements may be different and only partly overlapping with those processes that contribute to the development of a self-identification of handedness. The developmental processes of self-identified handedness may have more in common with those of self-identified gender, ethnicity, familial origins, and so on than with the control of the neural-motor control of the forelimbs.

Because all of these measures require that the individual follow instructions, they are not useful for investigations of infant handedness. Therefore, my collaborators and I (cf. Michel, 2002) have identified several early differences between the limbs in moving the arms (e.g., bringing the hand to the face and mouth), controlling the fingers of the hands (e.g., grasping and independent finger movement), swiping at visually presented objects, acquiring objects, unimanually manipulating objects, and role-differentiated bimanual manipulation (RDBM; one hand 
supporting the haptic exploration and manipulation of the object by the other hand). But are any or all of these hand differences in infancy equivalent to handedness in children and adults?

I proposed that infant handedness be defined as hand-use preferences for three common manual activities: acquiring objects, unimanual manipulation of objects, and RDBM of objects (the nonpreferred hand supports the manipulations of the object by the preferred hand). Each of these manual skills emerges during the infant's first year, eventually becoming integral components of all manual skills in the adult. The infant's preference in each of these activities reflects differences in the neural mechanisms involved in their sensorimotor control (Serrien, Ivry, \& Swinnen, 2006; Volkmann, Schnitzler, Witte, \& Freund, 1997). That is, the infant's "preference” depends upon differences in the control mechanisms for hand use (much like those involved in adult hand differences in speed and accuracy).

Although early forms of handedness use the same peripheral structure (the arm and the hand) for the manifestation of the behavior, are these nascent forms of handedness equivalent to those of adults? It is unlikely that the same neural circuits involved in the different forms of handedness examined at different developmental periods are equivalent. It is likely that there will be some overlap in circuits. However, actions that depend on precise timing of the serial order of muscle contractions for the successful execution of the manual action (e.g., RDBM, peg-moving) likely have a more extensive overlap in circuits than with those that do not (e.g., unimanual objectshaking/banging, finger tapping).

For example, some have proposed that evidence for the functional symmetry of the cerebral hemispheres is apparent as early as 10 to 15 weeks of fetal development (De Vries et al., 2001; Hepper, Shahidullah, \& White, 1991) when fetuses make unimanual contact with the face and may exhibit preferential arm movement. Indeed, Hepper, Wells, and Lynch (2005) reported that fetus's apparent differences in the movement patterns of the arms are predictive of later handedness, with $100 \%$ of fetuses with a prenatal preference to suck the thumb of the right hand exhibiting right-handedness (identified by parental report) at age 10 to 12 and $67 \%$ of fetuses who preferred to suck their left thumb exhibiting left-handedness. However, given the pattern of brain development, such early asymmetry of arm movements likely reflects the activation of spinal reflexes rather than either brain-stem or cortical circuits (cf. Hopkins \& Rönnqvist, 1998; Rönnqvist, Hopkins, van Emmerik, \& de Groot, 1998). Therefore, the mechanism by which such lateralized actions would be produced at 15 gestational weeks cannot involve the same cortical neural processes that are involved in handedness of children and adults.

Nevertheless, it is possible that, if such lateralized processes controlling limb actions existed at the level of the spinal cord, they could contribute to the developmental sculpting of the neural processes associated with further cerebral lateralization (brain stem, basal ganglia, limbic system, and cortex). Therefore, if fetal asymmetrical hand actions predict preteen handedness, then they must do so by contributing to the biasing of the development of the midbrain and forebrain mechanisms responsible for handedness in adults. 
We, also, consider the development of handedness to be based on intrauterine events. Asymmetries of fetal position and actions have been proposed to concatenate into the neonate's supine head orientation preference (HOP) (Fong, Savelsbergh, van Geijn, \& de Vries, 2005; Michel \& Goodwin, 1979; Previc, 1991). That is, for the first 2 months postpartum, an overwhelming majority of infants prefer to lie with their heads turned to their right and about 15\% prefer to turn their heads to the left (Michel, 1981). The fetus’ position during the last trimester likely contributed to the development of lateralized differences in the functioning of the vestibular system, the auditory system, and postnatal posture (Kurjak et al., 2004; Previc, 1991). It is likely that the direction of this HOP is a consequence of asymmetrical activation of neuromotor mechanisms (involving vestibular stimulation and reflexes) at the level of brain-stem nuclei, cerebellum, thalamus, and perhaps basal ganglia (Previc, 1991). Neonatal hand-use differences (e.g., grasping durations, arm movements, face touching, thumb sucking) depend on postural asymmetries associated with supine HOP (Michel, 2002). Although the mechanism controlling lateralized asymmetry in HOP is different from that controlling handedness in children and adults, they appear to be developmentally tied. The HOP influences early lateralized asymmetries of hand and arm actions and the HOP subsequently predicts development of rightand left-hand use preferences for reaching for, and acquiring, objects throughout their first 18 months (Michel, 1981; Michel \& Harkins, 1986).

Kupperstein (1988) proposed a mechanism by which the direction of HOP can affect the development of the infant's hand-use preference for acquiring objects. Visual fixation of the hand ("foveation") activates neural systems monitoring the tensions of the extraocular eye muscles; whereas, nonfoveated "looking” does not produce tensions in the eye muscles. During HOP, foveation of one hand occurs because the biased activation of the face-side arm-hand movements by the vestibular and neck stretch reflexes, prompted by the turned head, places the hand in a position that makes foveation more probable. Also, the face-side hand is precisely within the fixed focal length of the neonate's vision (van der Meer, van der Weel, \& Lee, 1995). An association is built between the activity of the extraocular muscles and that of the activity of muscles of the arm in their postural positions. Because the face-side hand is more active (Michel, 1981), it will attract foveation.

The neonatal HOP is maintained for the first 8 to 10 weeks postnatally and affects several differences between the hands via brain-stem mechanisms (Michel, 1981; Michel \& Goodwin, 1979; Michel \& Harkins, 1986). These differences promote differences between the face-side and skull-side hands that permit correspondent mapping in the nervous system of "felt" arm position, "seen” arm position, and "programmed” arm movements. This builds a proprioceptive "map" that combines head-eye position with hand-arm position and the hand's location in a "map" of visual space. Later in development, when an object is foveated, the correct arm muscle tensions are "recalled" so as to position the arm to move toward and contact the object.

Consequently, my collaborators and I have proposed that handedness development during infancy is a cascade of developmental contingencies with nascent forms of infant handedness 
contributing to the occurrence subsequent forms. The direction of the neonatal HOP predicts the hand preference for swiping at objects by age 4 months (Michel, 1981). These laterally asymmetrical swiping actions lead to hand-use preferences for reaching for and acquiring objects, including food (Michel \& Harkins, 1986). Throughout the 6- to 14-month-old period, reliable hand-use preferences are observed in reaching for and acquiring objects (Ferre, Babik, \& Michel, 2010), with many infants (38\%) exhibiting a consistent right hand-use preference and 14\% a consistent left hand-use preference (Michel, Babik, Sheu, \& Campbell, 2013). The remaining $48 \%$ of infants exhibit a significant linear developmental trend toward right handedness during this age period.

Also, during this age period, the hand-use preferences for acquiring objects subsequently spreads into hand-use preferences for unimanual manipulation of objects (Hinojosa, Sheu, \& Michel, 2003) and finally into hand-use preferences for RDBM and tool-use. In each instance of this spread of the preference, the infant exhibits the manual skill weeks or months before a hand-use preference appears. When that preference appears, it is predicted by the infant's preference for acquiring objects. Because of this longitudinal cascade of the manifestation of handedness, infant HOP can be predictive of infant hand-use preference in RDBM. Moreover, examination of these same infants' handedness for RDBM during the period from age 18 to 24 months reveals that those who had a consistent hand-use preference for acquiring objects at age 6 to 14 months exhibited the same hand-use preference in their pattern of RDBM (Nelson, Campbell, \& Michel, 2013). Those toddlers, who as infants did not manifest a consistent right hand-use preference, began manifesting a preference for RDBM with the majority exhibiting a right preference.

In addition to the effect of these self-generated experiences on the infant's handedness development, we have demonstrated that the handedness of the mother can facilitate and constrain the handedness development of her infant (Harkins \& Michel, 1988;Michel, 1992). Mothers unintentionally exhibit a bias in their play with their infants that encourages the use of the infant's hand that is concordant with their mother's handedness (Michel, 1992; Mundale, 1992). Thus, concordance of handedness between the mother and infant can facilitate the handedness of the infant; whereas, discordance of handedness can constrain the handedness development of the infant. The mother's handedness does not shape the direction of the handedness of her infant. Rather, the infant's handedness is less lateralized and takes longer to lateralize as a consequence of the discordance with the mother's handedness.

Thus, early handedness development appears to be a spreading cascade across different manual skills rather than simply an increase in hand preference within a skill. Michel (2002) proposed that by age 18 to 24 months, most children have a hand-use preference across a range of unimanual and bimanual skills that will form the basis for all future manual actions and hence their "handedness." Currently, we are charting the relation of the hand-use preferences in these infants and toddlers to their handedness, as measured by the standard peg-moving skills, during their third to fifth year of age. Such charting will provide evidence of the developmental relation of infant hand-use preferences to the handedness of children and perhaps adults. This detailed 
charting of the developmental trajectories for handedness can serve as a model for how developmental research on psychological traits should be conducted.

\section{CONCLUSIONS}

Developmental science must begin with a description of the trajectories for the development of phenotypic traits that permits incorporation of information about the physiological conditions and processes of the individual in interaction with the specific characteristics of the individual's social and physical milieu. DPB provides the training and expertise needed for such descriptions and for the conduct of such research.

\section{FUNDING}

Some of the research reported herein was supported by National Science Foundation grant DLS0718045 awarded to George F. Michel.

\section{REFERENCES}

1. Caspi, A., McClay, J., Moffitt, T. E., Mill, J., Martin, J., Craig, I. W. and Poulton, R. 2002. Role of genotype in the cycle of violence in maltreated children. Science, 297(5582): 851-854.

2. De Vries, J. I. P., Wimmers, R. H., Ververs, I. A. P., Hopkins, B., Savelsbergh, G. J. P. and Van Geijn, H. P. 2001. Fetal handedness and head position preference: A developmental study. Developmental Psychobiology, 39(3): 171-178.

3. Ferre, C. L., Babik, I. and Michel, G. F. 2010. Development of infant prehension handedness: a longitudinal analysis during the 6- to14-month age period. Infant Behavior and Development, 33(3): 492-502.

4. Fong, B. F., Savelsbergh, B. F., van Geijn, H. P. and de Vries, J. I. 2005. Does intra-uterine environment influence fetal head-position preference? A comparison between breech and cephalic presentation. Early Human Development, 81(6): 507-517.

5. Gilbert, S. F. and Epel, D. 2009. Ecological developmental biology, Sunderland, MA: Sinauer.

6. Gottlieb, G. 1992. Individual development and evolution: The genesis of novel behavior, New York, NY: Oxford University Press.

7. Gottlieb, G. 1999. Probabilistic epigenesis and evolution, Worcester, MA: Clark University Press.

8. Harkins, D. A. and Michel, G. F. 1988. Evidence for a maternal effect on infant handedness preferences. Developmental Psychobiology, 21: 535-54l. 
9. Hepper, P. G., Shahidullah, S. and White, R. 1991. Handedness in the human fetus. Neuropsychologia, 29: 1107-1111.

10. Hepper, P. G., Wells, D. L. and Lynch, C. 2005. Prenatal thumb sucking is related to postnatal handedness.Neuropsychologia, 43: 313-315.

11. Hinojosa, T., Sheu, C.-F. and Michel, G. F. 2003. Infant hand-use preferences for grasping objects contributes to the development of a hand-use preference for manipulating objects. Developmental Psychobiology, 43: 328-334.

12. Hopkins, B. and Rönnqvist, L. 1998. "Human handedness: Developmental and evolutionary perspectives". In The development of sensory, motor and cognitive capacities in early infancy: From sensation to cognition, Edited by: Simion, F. and Butterworth, G. E. 191-236. Hove, UK: Psychology Press. InEds.pp.

13. Jablonka, E. and Lamb, M. J. 2005. Evolution in four dimensions, Cambridge, MA: MIT Press.

14. Jaffee, S. R., Caspi, A., Moffitt, T. E., Dodge, K. A., Rutter, M., Taylor, A. and Tully, L. A. 2005. Nature x nurture: Genetic vulnerabilities interact with child maltreatment to promote conduct problems. Development and Psychopathology, 17: 67-84.

15. Kupperstein, M. 1988. Neural model of adaptive eye-hand coordination for single postures. Science, 239: 1308-1311.

16. Kurjak, A. M., Stanojevic, M., Andonotopo, W., Salihagic-Kadic, A., Carrera, J. M. and Azumendi, G. 2004. Behavioral pattern continuity from prenatal to postnatal life - a study by four-dimensional (4D) ultrasonography. Journal of Perinatal Medicine, 32(4): 346-353.

17. Michel, G. F. 1981. Right-handedness: A consequence of infant supine head-orientation preference?. Science, 212: 685-687.

18. Michel, G. F. 1992. Maternal influences on infant hand-use during play with toys. Behavior Genetics, 22: 163-176.

19. Michel, G. F. 2002. “Development of infant handedness”. In Conceptions of development, Edited by: Lewkowicz, D. J. and Lickliter, R. 165-186. New York, NY: Psychology Press. InEds.pp.

20. Michel, G. F. 2010a. Behavioral science, engineering and poetry revisited. Journal of Comparative Psychology, 124(3): 336-341.

21. Michel, G. F. 2010b. "The roles of environment, experience, and learning in behavioral development”. In Handbook of developmental science, behavior and genetics, Edited by: Hood, K., Halpern, C., Greenberg, G. and Lerner, R. 123-165. Malden, MA: Wiley. InEds.pp. 
22. Michel, G. F., Babik, I., Nelson, E. L., Campbell, J. M. and Marcinowski, E. C. 2013. How the development of handedness could contribute to the development of language. Developmental Psychobiology, 55(6): 608-620.

23. Michel, G. F., Babik, I., Sheu, C.-F. and Campbell, J. M. 2013. Latent classes in the developmental trajectories of infant handedness. Developmental Psychology, doi:10.1037/a0033312

24. Michel, G. F. and Goodwin, R. 1979. Intrauterine birth position predicts newborn supine head position preferences. Infant Behavior and Development, 2: 29-38.

25. Michel, G. F. and Harkins, D. A. 1986. Postural and lateral asymmetries in the ontogeny of handedness during infancy.Developmental Psychobiology, 19: 247-258.

26. Michel, G. F. and Moore, C. L. 1995. Developmental psychobiology: An interdisciplinary science, Cambridge, MA: MIT Press.

27. Michel, G. F., Nelson, E. L., Babik, I., Campbell, J. M. and Marcinowski, E. C. 2013. "Multiple trajectories in the developmental psychobiology of human handedness".

In Embodiment and epigenesis: Theoretical and methodological issues in understanding the role of biology within the relational developmental system part b: ontogenetic dimensions, Edited by: Lerner, R. M. and Benson, J. B. 227-260. New York, NY: Elsevier Inc.: Academic Press. InEds.pp.

28. Mundale, C. J. 1992. Influences of maternal handedness and behavior on infant hand use preferences, Psychology Department, DePaul University, Chicago, IL. (Master's Thesis)

29. Nelson, E. L., Campbell, J. M. and Michel, G. F. 2013. Unimanual to bimanual: Tracking the development of handedness from 6 to 24 months. Infant Behavior and Development, 36: 181188.

30. Previc, E. H. 1991. A general theory concerning the prenatal origins of cerebral lateralization in humans. Psychological Review, 98: 299-334.

31. Rönnqvist, L., Hopkins, B., van Emmerik, R. and de Groot, L. 1998. Lateral biases in head turning and the Moro response in the human newborn: are they both vestibular in origin?. Developmental Psychobiology, 33: 339-349.

32. Serrien, D. J., Ivry, R. B. and Swinnen, S. P. 2006. Dynamics of hemispheric specialization and integration in the context of motor control. Nature Reviews Neuroscience, 7(2): 160-167.

33. Sperry, R. W. 1965. "Mind, brain and humanist values". In New views of the nature of man, Edited by: Platt, J. R. 71-92. Chicago, IL: University of Chicago Press. InEd.pp. 
34. Tucker-Drob, E. M., Briley, D. A. and Harden, K. P. 2013. Genetic and environmental influences on cognition across development and context. Current Directions in Psychological Science, 22: 349-355.

35. Van der Meer, A. L. H., van der Weel, F. R. and Lee, D. N. 1995. The functional significance of arm movements in neonates.Science, 267: 693-695.

36. Volkmann, J., Schnitzler, A., Witte, O. W. and Freund, H.-J. 1997. Handedness and asymmetry of hand representation in human motor cortex. Journal of Neurophysiology, 79(4): 2149-2154. 